\title{
Effect of temperature on thromboelastography and implications for clinical use in newborns undergoing therapeutic hypothermia
}

\author{
Katie R. Forman ${ }^{1,2}$, Edward Wong ${ }^{2,3,4}$, Meanavy Gallagher ${ }^{3}$, Robert McCarter ${ }^{2,5,6}$, Naomi L.C. Luban $^{2,3,4}$ and An N. Massaro ${ }^{1,2}$
}

BACKGROUND: Encephalopathic neonates undergoing therapeutic hypothermia have increased risk for coagulopathy secondary to perinatal asphyxia and effects of cooling on the coagulation enzyme cascade. Thromboelastography (TEG) allows for a comprehensive assessment of coagulation that can be regulated for temperature. TEG has not been previously evaluated in newborns undergoing hypothermia treatment.

METHODS: Encephalopathic neonates treated with systemic hypothermia were enrolled in this prospective observational study. Daily blood specimens were collected for standard coagulation tests and platelet counts during hypothermia and after rewarming. Concurrent TEG assays were performed at 33.5 and $37.0^{\circ} \mathrm{C}$ for comparison.

RESULTS: A total of 48 paired TEGs from 24 subjects were performed. Forty percent of the subjects were males, the mean ( \pm SD) birth weight was $3.2 \pm 0.7 \mathrm{~kg}$, and the mean gestational age was $38.4 \pm 1.4 \mathrm{wk}$. TEG results differed significantly between assays performed at 37.0 vs. $33.5^{\circ} \mathrm{C}$, indicating more impaired coagulation at $33.5^{\circ} \mathrm{C}$. TEG parameters clot kinetics, angle, maximum amplitude (MA), and coagulation index were significantly associated with clinical bleeding $(P<0.05)$. These remained significant (except for $\mathrm{MA}$ ) after controlling for transfusion therapy.

CONCLUSION: TEG results are affected by temperature, consistent with the known association of hypothermia with coagulopathy. Several TEG parameters are predictive of clinical bleeding in newborns undergoing hypothermia. Selected cutpoints to predict bleeding risk are temperature dependent.

$\mathbf{N}$ eonates with hypoxic ischemic encephalopathy are at increased risk for coagulopathy $(1,2)$. Systemic oxygen deprivation impacting the liver and bone marrow impairs the synthesis of coagulation factors and platelets (3-6). It is also well established that a hypothermic environment exacerbates coagulation disturbances by decreasing enzymatic activity involved in the coagulation cascade (7-12). This phenomenon is increasingly important because therapeutic hypothermia is the current standard of care for infants affected by hypoxic ischemic encephalopathy (13-22).

A wide variability exists among clinicians and institutions with regards to protocols for monitoring coagulation status in newborns undergoing therapeutic hypothermia. This is, in part, due to the uncertainty of whether transfusion therapy should target normalization of standard tests of coagulation vs. a more conservative approach of initiating treatment only after clinical bleeding is observed. Algorithms to optimize transfusion therapy to be able to prevent clinical bleeding while minimizing exposure to excessive blood products are lacking. One difficulty arises from the fact that standard coagulation tests (i.e., activated partial thromboplastin time (aPTT), prothrombin time, and international normalized ratio (INR)) are routinely performed at $37.0^{\circ} \mathrm{C}(8)$. This may not accurately reflect the in vivo condition of a patient undergoing moderate hypothermia who is maintained at a core temperature of $33.5^{\circ} \mathrm{C}(17)$. Alternative methods to assess coagulation status in this population are needed.

Thromboelastography (TEG) is a functional assay that evaluates the process of clot formation and degradation in a whole blood sample (23-26). In a single test, TEG comprehensively reflects the complex clotting process that involves coagulation factors, cellular components, enzymes, and highly organized feedback mechanisms that maintain equilibrium between clot formation and lysis (1). TEG can be easily calibrated to the temperature of the patient. Despite this advantage that is particularly attractive in neonates undergoing hypothermia, TEG has not been previously evaluated in this population at high risk for coagulopathy.

The aim of this study is to evaluate the utility of TEG in newborns undergoing therapeutic hypothermia. We hypothesized that TEG would demonstrate quantifiable differences when performed under normothermic $\left(37.0^{\circ} \mathrm{C}\right)$ vs. hypothermic $\left(33.5^{\circ} \mathrm{C}\right)$ conditions. Secondarily, we hypothesized that TEG performed at $33.5^{\circ} \mathrm{C}$ would predict clinical bleeding in this high-risk neonatal population. 


\section{Articles $\mid$ Forman et al.}

\section{RESULTS}

\section{Study Population Characteristics}

A total of 24 patients were enrolled and received treatment with therapeutic hypothermia either for hypoxic ischemic encephalopathy $(n=22)$ or for hyperammonemia $(n=2)$. Characteristics of the study population are summarized in Table 1. Of the 24 patients enrolled, 17 patients demonstrated a total of 27 bleeding events. These events included pulmonary hemorrhage ( $n=14)$, gastrointestinal bleeding $(n=17)$, hematuria $(n=3)$, and mucosal (i.e., profuse umbilical stump and arterial line site) bleeding $(n=2)$.

\section{Effect of Temperature on TEG}

A total of 48 paired TEGs were performed at 33.5 and 37.0 ${ }^{\circ} \mathrm{C}$. Bivariate analyses demonstrated significant differences in TEG clotting time $(R)$, clot kinetics $(K)$, angle $(\alpha)$, and coagulation index $(\mathrm{CI})(P<0.01$; Figure 1$)$. Differences in each TEG measure indicated more impaired coagulation in the assays performed at $33.5^{\circ} \mathrm{C}$. These differences remained significant for TEG $K(\beta=-0.148$; $\mathrm{SE}=0.068$; $95 \%$ confidence interval: 0.281 to $-0.013 ; P=0.031)$ and $\alpha(\beta=1.167 ; \mathrm{SE}=0.371 ; 95 \%$ confidence interval: $0.440-1.894 ; P=0.002)$ in the multivariate longitudinal regression models.

\section{TEG to Predict Bleeding}

Several TEG parameters including $K, \alpha$, maximum amplitude (MA), and CI demonstrated an association with clinical bleeding $(P<0.05)$. These associations were significant for $K, \alpha$, and $\mathrm{CI}$ at $37.0^{\circ} \mathrm{C}$ and $\alpha$ at $33.5^{\circ} \mathrm{C}$ after controlling for transfusion

Table 1. Clinical and demographic characteristics of study population

\begin{tabular}{|c|c|c|}
\hline & $\operatorname{HIE}(n=22)$ & Hyperammonemia $(n=2)$ \\
\hline Birth weight $(\mathrm{g})^{\mathrm{a}}$ & $3,273 \pm 699$ & $3,256 \pm 65$ \\
\hline Gestational age (wk) ${ }^{\mathrm{a}}$ & $38.74 \pm 1.36$ & $38.65 \pm 0.92$ \\
\hline Male gender, $n(\%)$ & $11(50)$ & $0(0)$ \\
\hline Cesarean delivery, $n(\%)$ & $19(86)$ & $1(50)$ \\
\hline Sentinel event, $n(\%)$ & $7(32)$ & $\mathrm{N} / \mathrm{A}$ \\
\hline Abruption, $n(\%)$ & $3(42.8)$ & \\
\hline Cord accident, $n(\%)$ & $3(42.8)$ & \\
\hline Maternal collapse, $n(\%)$ & $1(14.2)$ & \\
\hline \multicolumn{3}{|l|}{ Apgar at } \\
\hline $1 \mathrm{~min}$ & $1(1,5)$ & $8(8)$ \\
\hline $5 \mathrm{~min}$ & $3(1,9)$ & $9(9)$ \\
\hline $10 \mathrm{~min}$ & $4(2,7)$ & $\mathrm{N} / \mathrm{A}$ \\
\hline Presenting $\mathrm{pH}$ & $7.01(6.65,7.35)$ & $\mathrm{N} / \mathrm{A}$ \\
\hline Base deficit & $-16(-34,-6)$ & N/A \\
\hline $\begin{array}{l}\text { Hour of life hypothermia } \\
\text { initiated (h:min) }\end{array}$ & $4: 40(2: 4,5: 58)$ & $89: 50(28: 54,120: 45)$ \\
\hline EEG seizure, $n(\%)$ & $4(18.2)$ & $0(0)$ \\
\hline
\end{tabular}

Data are presented as median (range) except where indicated. aMean \pm SD.

EEG, electroencephalogram; HIE, hypoxic ischemic encephalopathy; N/A, not applicable. therapy. Logistic regression model results are summarized in Table 2. aPTT $(P=0.961)$, INR $(P=0.363)$, and platelet count $(P=0.718)$ were not associated with clinical bleeding. Receiver operator curves demonstrated significant area under the curve for TEG $K, \alpha$, and MA at both 33.5 and $37.0^{\circ} \mathrm{C}$, and $\mathrm{CI}$ at $33.5^{\circ} \mathrm{C}$ (Figure 2). While receiver operator curves were significant at both temperatures, the selected cutpoint differed based on temperature.

\section{DISCUSSION}

This is the first study conducted to evaluate the effect of temperature on individual TEG parameters and to evaluate the potential utility of TEG to monitor coagulation status in newborns undergoing therapeutic hypothermia. These data demonstrate quantifiable differences in coagulation status based on temperature, with more impaired coagulation under hypothermic conditions. Thus, standard tests of coagulation performed on blood warmed to $37.0^{\circ} \mathrm{C}$ may underestimate coagulopathy in patients who are undergoing hypothermia. An association with clinical bleeding could be demonstrated with several TEG parameters, whereas standard tests of coagulation (aPTT and INR) were not associated with bleeding. This provides further evidence that TEG may serve as the preferred method of monitoring coagulation status in this population.

Although TEG has been routinely utilized in adult populations for the past $20 \mathrm{y}(24-26)$, it has only recently emerged for use in pediatrics. Early reports have demonstrated its utility in monitoring coagulation status in children requiring cardiac bypass surgery, liver transplantation, neurosurgery, or extracorporeal membrane oxygenation (27-31). While normative data are available for pediatric patients (32), there are limited data reported in neonates. Only one study reported references ranges for newborns based on umbilical cord blood specimens (23). However, it is unknown whether these values represent normative values for neonates in the first days of life postpartum. The data provided in this study support that further evaluation in neonatal populations, particularly in critically ill neonates at high risk for coagulopathy, is warranted. An advantage of TEG is the ease of calibration for temperature, which is of particular interest in patients undergoing hypothermia. The two variables most influenced by temperature were $K$ and $\alpha$. These two parameters are the most reflective of clotting factor and enzymatic activity. Under hypothermic conditions, clot kinetics were slower, indicating a delay in enzymatic activity, and $\alpha$ was narrower, indicating lower efficiency in attaining maximum clot strength. Other TEG variables, including clot reaction time and $\mathrm{CI}$, also trended in a direction consistent with the hypothesis that hypothermia slows enzymatic activity and impairs the coagulation cascade. Of interest, MA, which reflects platelet activity, did not vary based on temperature although there is known cold inhibition on platelet function (33). It should be noted that TEG may be insensitive to mild qualitative platelet dysfunction and likely more sensitive to platelet count (i.e., thrombocytopenia). For example, effects of aspirin and clopidigrel used in Berlin Heart protocols were not readily reflected with regular TEG and required platelet 
a

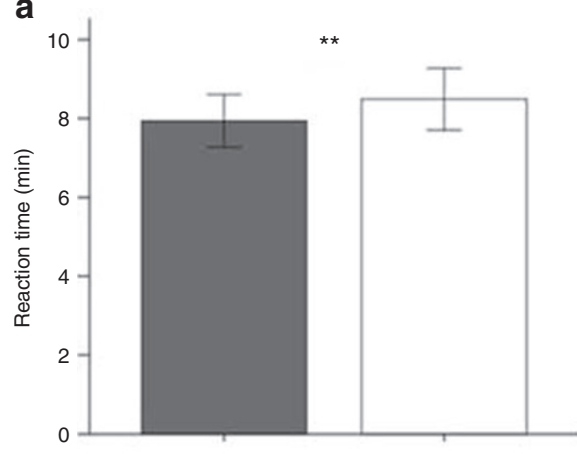

d

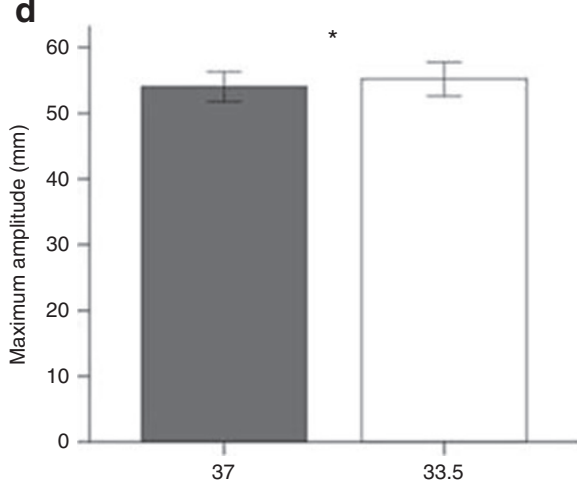

b
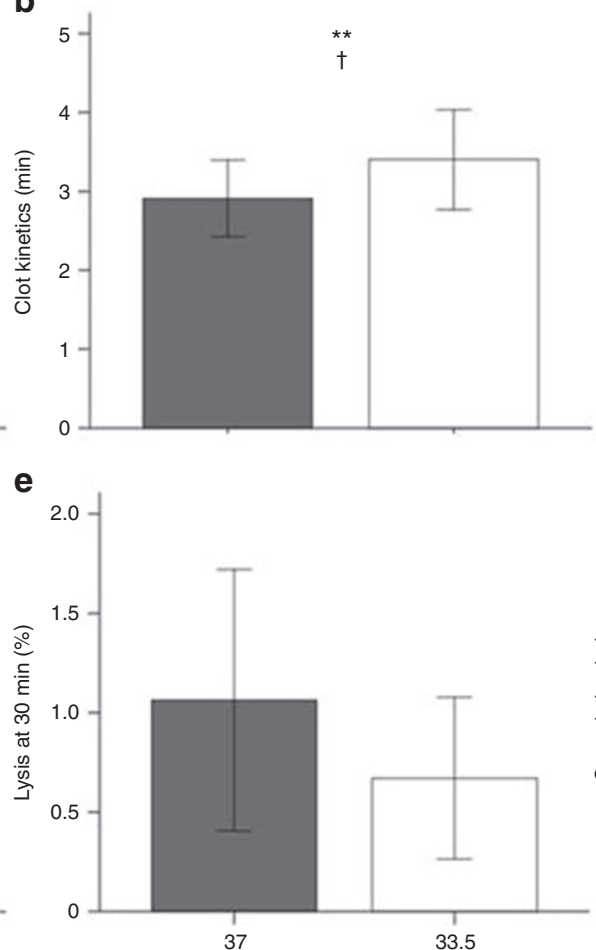

C

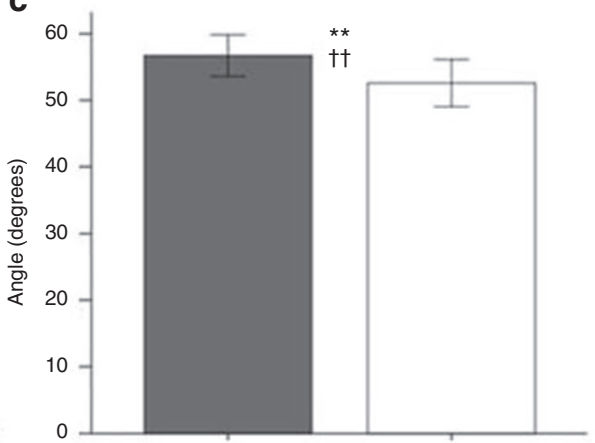

f

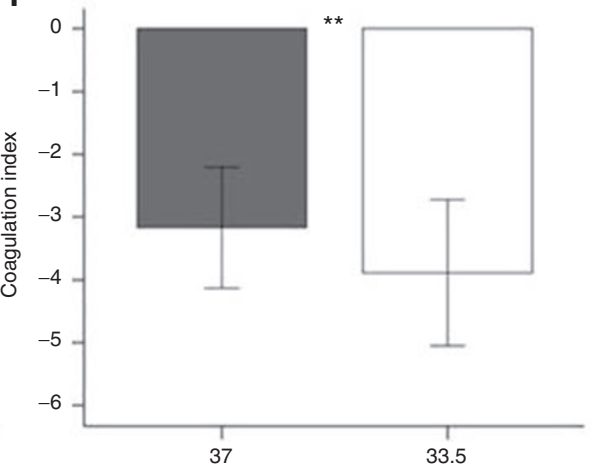

Figure 1. Effect of temperature on thromboelastography parameters. (a) Reaction time in minutes, (b) clot kinetics in minutes, (c) angle in degrees, (d) maximum amplitude in mm, (e) lysis at 30 minutes as a percent, and (f) coagulation index. The gray bars represent $37^{\circ} \mathrm{C}$, whereas the white bars represent $33.5^{\circ} \mathrm{C}$. All bars represent mean $\pm 95 \%$ confidence interval. Significant differences by paired $t$-tests are shown with asterisks $\left({ }^{*} P<0.05\right.$, $\left.{ }^{*} P<0.001\right)$. Significant adjusted $P$ values are marked with daggers $\left({ }^{\dagger} P<0.05,{ }^{+\dagger} P<0.01\right)$.

Table 2. Summary of regression analysis results evaluating the association of TEG with clinical bleeding

\begin{tabular}{cccccc}
\hline & $\begin{array}{c}\text { TEG } \\
\text { parameter }\end{array}$ & $\begin{array}{c}\text { Adjusted } \\
\text { odds ratio }\end{array}$ & SE & $\begin{array}{c}\text { 95\% Confidence } \\
\text { interval }\end{array}$ & $P$ value \\
\hline $37^{\circ} \mathrm{C}$ & $R$ & 1.075 & 0.273 & $0.654-1.769$ & 0.773 \\
& $K$ & 4.156 & 2.837 & $1.09-15.845$ & 0.037 \\
& $\alpha$ & 0.849 & 0.062 & $0.737-0.979$ & 0.025 \\
& $\mathrm{MA}$ & 0.770 & 0.110 & $0.582-1.019$ & 0.067 \\
$33.5^{\circ} \mathrm{C}$ & $\mathrm{Cl}$ & 0.637 & 0.146 & $0.407-0.999$ & 0.049 \\
& $\mathrm{LY} 30$ & 0.832 & 0.261 & $0.450-1.540$ & 0.559 \\
& $K$ & 1.056 & 0.224 & $0.696-1.601$ & 0.798 \\
& $K$ & 2.123 & 0.849 & $0.970-4.649$ & 0.060 \\
& $\alpha$ & 0.879 & 0.056 & $0.775-0.996$ & 0.044 \\
& $\mathrm{MA}$ & 0.781 & 0.102 & $0.605-1.007$ & 0.057 \\
& $\mathrm{Cl}$ & 0.732 & 0.101 & $0.521-1.027$ & 0.071 \\
& $\mathrm{LY} 30$ & 0.392 & 0.348 & $0.691-2.228$ & 0.291 \\
\hline
\end{tabular}

$\alpha$, Angle; $\mathrm{Cl}$, coagulation index; $K$, clot kinetics; $\mathrm{MA}$, maximum amplitude; $R$, clotting time; TEG, thromboelastography.

mapping (34). It is not surprising that LY30 did not appear to vary based on temperature as this variable is reflective of a process less dependent on initial enzymatic activity (i.e., LY30 measures clot stability).

Previous multicenter cooling trials did not report increased rates of major hemorrhage or bleeding-related deaths in patients undergoing this therapy. However, consistent findings across studies include thrombocytopenia and prolonged prothrombin time/aPTT likely associated with the need for transfusion therapy to prevent bleeding risk $(13,17-$ 22). Overall rates of clinical bleeding requiring intervention are not well described by prior studies. Our study suggests that these events may occur frequently in this population. The definition of clinically relevant bleeding used in our study was based on recommendations by the International Society of Thrombosis and Haemostasis (35). While the bleeding events demonstrated in patients enrolled in this study were not life threatening in nature, they all required transfusion therapy or other active intervention (e.g., increase in positive end expiratory pressure in cases of pulmonary hemorrhage). It is unclear whether transfusion therapy should be targeted to normalizing standard tests of coagulation or conservatively initiating therapy only after clinical bleeding is exhibited. Data from this study suggest that the former approach is problematic given the poor discriminatory ability of standard tests of coagulation to predict clinical bleeding. In an ideal setting, transfusion therapy should be targeted to a specific product administered with an appropriate dose to prevent any bleeding. This study provides evidence that TEG may provide a better method to monitor bleeding risk and provide guidance for rational transfusion therapy.

Another benefit of TEG is that it provides a global measure of coagulation in a single test, utilizing a small volume of blood $(0.36 \mathrm{ml})$. This is of particular interest in the critically ill neonatal population given that the frequency of blood sampling 

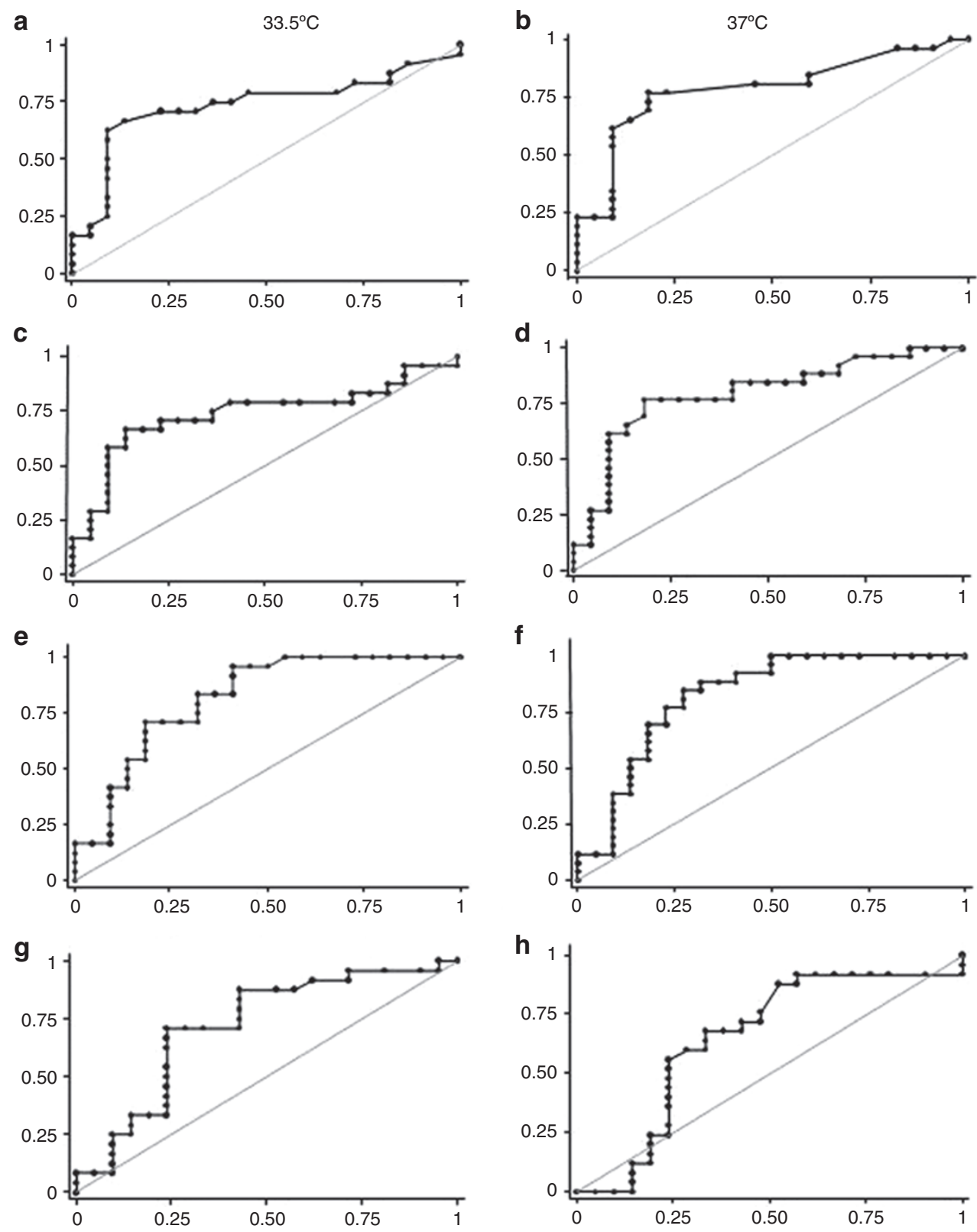

Figure 2. Receiver operating curves for clot kinetics $(K)$, angle $(\alpha)$, maximum amplitude $(\mathrm{MA})$, and coagulation index $(\mathrm{Cl})$. The diagonal line represents an area under the curve (AUC) of 0.5 , indicating a noninformative test. (a) $\mathrm{K}$ performed at $33.5^{\circ} \mathrm{C}$. AUC $=0.7377$, cutpoint $=3.4$ min, sensitivity $=62.5 \%$, and specificity $=90.9 \%$. (b) $\mathrm{K}$ performed at $37^{\circ} \mathrm{C}$. AUC $=0.7876$, cutpoint $=2.6 \mathrm{~min}$, sensitivity $=76.9 \%$, and specificity $=81.8 \%$. (c) $\alpha$ Performed at $33.5^{\circ} \mathrm{C}$. AUC $=07377$, cutpoint $=52.3$ degrees, sensitivity $=66.7 \%$, and specificity $=88.4 \%$. (d) $\alpha$ Performed at $37^{\circ} \mathrm{C}$. AUC $=0.7955$, cutpoint $=57.6$ degrees, sensitivity $=76.9 \%$, and specificity $=81.8 \%$. (e) MA performed at $33.5^{\circ} \mathrm{C}$. AUC $=0.8172$, cutpoint $=56.1 \mathrm{~mm}$, sensitivity $=70.83 \%$, and specificity $=81.82$. (f) $\mathrm{MA}$ performed at $37^{\circ} \mathrm{C}$. AUC $=0.8217$, cutpoint $=58.1 \mathrm{~mm}$, sensitivity $=88.46 \%$, specificity $=61.18 \%$. (g) $\mathrm{Cl}$ performed at $33.5^{\circ} \mathrm{C}$. AUC $=0.7173$, cutpoint $=-4.6$, sensitivity $=70.83 \%$, and specificity $=76.19 \%$. (h) Cl performed at $37^{\circ} \mathrm{C}$. AUC $=0.6476$, cutpoint $=-3.3$, sensitivity $=68 \%$, and specificity $=66.67 \%$.

is often problematic and can lead to iatrogenic anemia. Each of the current standard tests used to assess coagulation status (prothrombin time, aPTT, INR, fibrinogen and platelet count) evaluates one aspect of a complex clotting process. These are often ordered and interpreted as a battery of tests that may be replaceable with the information provided by TEG.

TEG parameters at both 33.5 and $37.0^{\circ} \mathrm{C}$ were found to be predictive of clinical bleeding. Of great importance, however, is the observation of different cutpoints to predict bleeding at the two temperatures. For example, the cutpoint for TEG
$K$ performed at $37^{\circ} \mathrm{C}$ is $2.6 \mathrm{~min}$, which actually falls within the normal adult range for this parameter. In contrast, when the assay is performed at $33.5^{\circ} \mathrm{C}$ (i.e., reflecting the hypothermic environment that the patient is experiencing), the cutpoint for TEG $K$ is higher at $3.4 \mathrm{~min}$. Thus, for accurate interpretation, TEG assays should be performed under temperature-regulated conditions (i.e., at $33.5^{\circ} \mathrm{C}$ for patients undergoing therapeutic hypothermia). Depending on institutional or laboratory preferences, an alternative strategy would include performing TEG under normothermic conditions and using different 
interpretive thresholds as provided in this study. Regardless, it is notable that several TEG parameters were more predictive of clinical bleeding than aPTT and INR.

This study has limitations. Although repeated measures provided improved power for statistical analyses, the small number of patients included may have limited our ability to achieve statistical significance where statistical trends were observed. Thus, the association between TEG values and clinical bleeding warrants investigation in a larger population. Sample size limitations also affected our ability to evaluate if these relationships differed between the patients cooled for neonatal encephalopathy vs. hyperammonemia. Although animal evidence suggests that elevated ammonia may impact platelet function directly (36), the coagulation disturbances in this population are likely attributable to the association of hyperammonemia with transaminitis and liver dysfunction reflected by a decrease in hepatic synthetic function (37). These anomalies would be revealed in TEG measures as described. We attempted to define "clinically significant" bleeding events. Commonly, iatrogenic trauma from nasogastric tube placement can lead to bloody gastric residuals, traumatic intubation can lead to bloody endotracheal tube secretions, and urinary catheter placement can lead to gross hematuria. These bleeding events may occur without underlying coagulopathy. These types of bleeding events were excluded unless they were observed repeatedly and met the defined criteria for clinically significant bleeding (i.e., decreased hemoglobin by $2 \mathrm{~g} / \mathrm{dl}$ in $24 \mathrm{~h}$, bleeding that required blood products for hemostasis or was found in critical organ). Due to obvious feasibility limitations, we were unable to obtain TEG data from healthy newborns for comparison. As previously discussed, normative data for TEG parameters in peripheral blood specimens from neonatal patients are lacking. Thus, the proposed cutpoints to predict bleeding cannot be interpreted in comparison to normative values for neonates.

\section{Conclusion}

TEG clot kinetics and angle are altered by temperature, providing a method to quantify impaired coagulation under hypothermic conditions. Several TEG parameters $(K, \alpha, \mathrm{MA}$, and
CI) are predictive of clinical bleeding in newborns undergoing therapeutic hypothermia, whereas current standard measurements of coagulopathy (aPTT and INR) were not predictive of bleeding. TEG may be a preferable way to monitor bleeding risk and guide transfusion therapy in this high-risk neonatal population.

\section{METHODS}

\section{Study Population}

This prospective observational study was conducted at an outborn level 4 neonatal intensive care unit in an academic free-standing children's hospital. All patients meeting the established criteria for treatment with hypothermia from August 2011 to July 2012 were approached for enrollment. An additional two patients with encephalopathy secondary to hyperammonemia were treated with hypothermia under an experimental protocol, and these two patients were also included in the study. All patients underwent whole-body therapeutic hypothermia according to the National Institute of Child Health and Human Development Neonatal Research Network protocol (17). Patients were cooled to an esophageal temperature of $33.5^{\circ} \mathrm{C}$ with a servo-regulated Blanketrol II (Cincinnati Sub-Zero Medical, Cincinnati, $\mathrm{OH}$ ) cooling blanket. Verbal consent was obtained from the parent(s) of each participant, and the need for written documentation of informed consent was waived for this minimal risk study. The Children's National Medical Center Institutional Review Board approved the study, and all data were collected in compliance with the Health Information Portability and Accountability Act regulations.

\section{Data Collection}

Demographic data (including gestational age, birth weight, and gender) and clinical data (including presenting characteristics, bleeding, and transfusion data) were prospectively collected after enrollment. Significant bleeding was defined as clinically overt bleeding (e.g., pulmonary hemorrhage, gastrointestinal bleeding, gross hematuria, or mucosal bleeding) associated with a decreased hemoglobin level by $2 \mathrm{~g} / \mathrm{dl}$ in a 24 -h period, bleeding that required blood products or surgical intervention for hemostasis or bleeding that was found in critical organ systems (i.e., intracranial, pulmonary, or retroperitoneal) (35). Bleeding data were noted for the 24 -h period following each TEG determination. Transfusions with fresh frozen plasma, cryoprecipitate, and/or platelets for each corresponding time period were also recorded.

\section{Specimen Collection and Processing}

Standard coagulation tests are routinely monitored daily during hypothermia and after rewarming per the Children's National Medical Center neonatal intensive care unit protocol. Up to three serial TEGs were performed per subject at $0-24,24-48$, and $48-72 \mathrm{~h}$ of cooling.

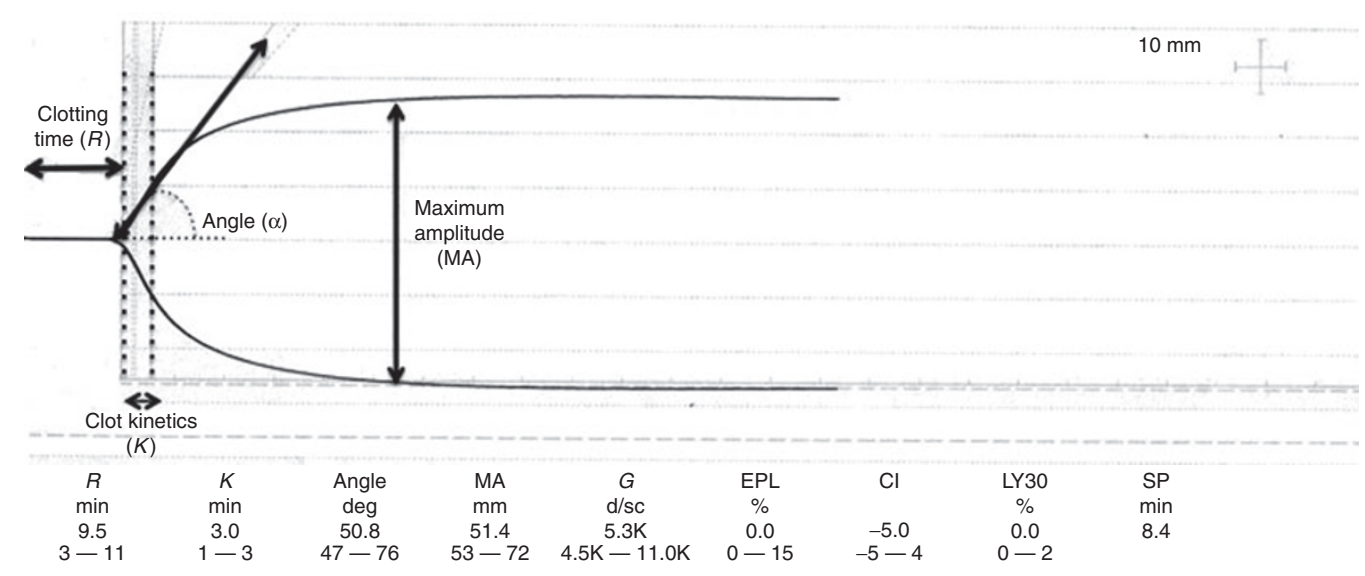

Figure 3. Representative example of thromboelastograph with measured parameters depicted schematically. Cl, coagulation index; EPL, estimated percent lysis; G, clot strength; MA, maximum amplitude; SP, split point. 
Whole blood specimens $(2.7 \mathrm{ml})$ were collected from indwelling arterial lines into vials containing $0.3 \mathrm{ml}$ of $3.2 \%$ sodium citrate. One milliliter of blood was removed for TEG analysis, whereas the remainder was used for clinically indicated prothrombin time, activated partial thromboplastin time (APTT), and fibrinogen assays (using the STA-R Evolution coagulation analyzer (Diagnostica STAGO, Parsippany, NJ) per manufacturer's instructions). A separate specimen was simultaneously collected to perform routine complete blood counts using the XE-5000 (Sysmex USA, Mundelein, IL). Specimens were hand delivered to the central laboratory for processing within $30 \mathrm{~min}$ of collection.

\section{TEG Determinations}

TEG determinations were performed on the dual-channel Haemoscope's TEG 5000 analyzer (Haemonetics, Braintree, MA) after kaolin activation according to the manufacturer's instructions. Briefly, $0.36 \mathrm{ml}$ of kaolin-activated, citrated whole blood is placed in a heparinase cup that is rotated $4^{\circ} 45^{\prime}$ six times per minute to imitate sluggish venous flow and activate the clotting cascade. A pin is suspended within the sample, and as fibrin clot forms between the wall of the cup and the pin, the pin is displaced in synchrony with the cup. The transmitted rotation from the cup to the pin is transduced into a computer analyzer that produces a tracing called a thromboelastograph (Figure 3 ). The four variables directly measured are (i) clotting time $(R)$ : the time of latency until initial fibrin formation, (ii) clot kinetics $(K)$ : the speed at which a specific level of clot strength is achieved (20-mm amplitude), (iii) angle $(\alpha)$ : the rapidity of clot strengthening, and (iv) maximum amplitude (MA): the ultimate strength of the clot. Additionally, a CI is derived from the four directly measured variables. Finally, the LY30 measures the rate at which the amplitude decreases $30 \mathrm{~min}$ after MA is achieved and is representative of clot breakdown or fibrinolysis. Therefore, impaired coagulation is reflected by a higher $R, K, \mathrm{LY} 30$ and/or lower MA, $\alpha, \mathrm{CI}$ $(23,24)$. One channel of the TEG analyzer was calibrated to $37.0^{\circ} \mathrm{C}$ and the second to $33.5^{\circ} \mathrm{C}$ for simultaneous determination of TEG under normothermic and hypothermic conditions. Six quantitative measures from the TEG trace $(R, K, \alpha, \mathrm{MA}, \mathrm{CI}$, and LY30) were recorded for each paired TEG. To minimize channel bias, temperature settings of each channel were alternated with each new specimen.

\section{Statistical Analysis}

Descriptive statistics include mean ( $\pm 95 \%$ confidence intervals) or median (range) for parametric and nonparametric continuous variables, respectively. Categorical variables were expressed as frequencies. Bivariate analyses were performed to assess the effect of temperature on each of the six parameters measured by TEG $(R, K$, $\alpha$, MA, CI, and LY30) with paired-samples $t$-tests. To account for intrasubject correlation due to repeated measurements in each subject, temperature effect was further evaluated by a random effects longitudinal regression model with covariables including day of measurement and baseline coagulation status (defined by INR obtained on admission to the neonatal intensive care unit). Logistic regression models were used to explore the role of TEG (and standard tests of coagulation aPTT and INR) to predict clinical bleeding controlling for transfusion therapy. Parameters that were associated with bleeding were further evaluated using receiver operator curve analyses in which an area under the curve of 1 denotes 100\% agreement between predicted and actual outcomes, whereas an area under the curve of 0.5 signifies no significant model discrimination. Coordinates of the receiver operator curves with significant area under the curves were used to determine cutpoints with optimal sensitivity and specificity to predict clinical bleeding.

\section{ACKNOWLEDGMENTS}

The authors acknowledge Jianping (James) He, for his data management and statistical support.

\section{STATEMENT OF FINANCIAL SUPPORT}

This project was financially supported by the Haemonetics Corporation (Braintree, MA). The sponsors had no role in the design and conduct of the study; in the collection, management, analysis, and interpretation of data; or in the preparation, review, or approval of the manuscript.

Disclosure: The authors have no financial relationships relevant to this article to disclose.

\section{REFERENCES}

1. Bauman ME, Cheung PY, Massicotte MP. Hemostasis and platelet dysfunction in asphyxiated neonates. J Pediatr 2011;158:Suppl:e35-9.

2. Suzuki S, Morishita S. Hypercoagulability and DIC in high-risk infants. Semin Thromb Hemost 1998;24:463-6.

3. Shah P, Riphagen S, Beyene J, Perlman M. Multiorgan dysfunction in infants with post-asphyxial hypoxic-ischaemic encephalopathy. Arch Dis Child Fetal Neonatal Ed 2004;89:F152-5.

4. Sarkar S, Barks JD, Bhagat I, Donn SM. Effects of therapeutic hypothermia on multiorgan dysfunction in asphyxiated newborns: whole-body cooling versus selective head cooling. J Perinatol 2009;29:558-63.

5. Castle V, Andrew M, Kelton J, Giron D, Johnston M, Carter C. Frequency and mechanism of neonatal thrombocytopenia. J Pediatr 1986;108(5 Pt 1):749-55.

6. Roberts IA, Murray NA. Thrombocytopenia in the newborn. Curr Opin Pediatr 2003;15:17-23.

7. Michelson AD, Barnard MR, Khuri SF, Rohrer MJ, MacGregor H, Valeri CR. The effects of aspirin and hypothermia on platelet function in vivo. Br J Haematol 1999;104:64-8.

8. Reed RL 2nd, Johnson TD, Hudson JD, Fischer RP. The disparity between hypothermic coagulopathy and clotting studies. J Trauma 1992;33:465-70.

9. Reed RL 2nd, Bracey AW Jr, Hudson JD, Miller TA, Fischer RP. Hypothermia and blood coagulation: dissociation between enzyme activity and clotting factor levels. Circ Shock 1990;32:141-52.

10. Rohrer MJ, Natale AM. Effect of hypothermia on the coagulation cascade. Crit Care Med 1992;20:1402-5.

11. Straub A, Breuer M, Wendel HP, Peter K, Dietz K, Ziemer G. Critical temperature ranges of hypothermia-induced platelet activation: possible implications for cooling patients in cardiac surgery. Thromb Haemost 2007;97:608-16.

12. Wolberg AS, Meng ZH, Monroe DM 3rd, Hoffman M. A systematic evaluation of the effect of temperature on coagulation enzyme activity and platelet function. J Trauma 2004;56:1221-8.

13. Jacobs SE, Berg M, Hunt R, Tarnow-Mordi WO, Inder TE, Davis PG. Cooling for newborns with hypoxic ischaemic encephalopathy. Cochrane Database Syst Rev 2013;1:CD003311.

14. Shankaran S. Therapeutic hypothermia for neonatal encephalopathy. Curr Treat Options Neurol 2012;14:608-19.

15. Shankaran S, Pappas A, McDonald SA, et al.; Eunice Kennedy Shriver NICHD Neonatal Research Network. Childhood outcomes after hypothermia for neonatal encephalopathy. N Engl J Med 2012;366:2085-92.

16. Shankaran S, Laptook AR, Tyson JE, et al.; Eunice Kennedy Shriver National Institute of Child Health and Human Development Neonatal Research Network. Evolution of encephalopathy during whole body hypothermia for neonatal hypoxic-ischemic encephalopathy. J Pediatr 2012;160:567-572.e3.

17. Shankaran S, Laptook AR, Ehrenkranz RA, et al.; National Institute of Child Health and Human Development Neonatal Research Network. Whole-body hypothermia for neonates with hypoxic-ischemic encephalopathy. N Engl J Med 2005;353:1574-84.

18. Azzopardi D, Brocklehurst P, Edwards D, et al.; TOBY Study Group. The TOBY Study. Whole body hypothermia for the treatment of perinatal asphyxial encephalopathy: a randomised controlled trial. BMC Pediatr 2008;8:17.

19. Gunn AJ, Wyatt JS, Whitelaw A, et al.; CoolCap Study Group. Therapeutic hypothermia changes the prognostic value of clinical evaluation of neonatal encephalopathy. J Pediatr 2008;152:55-8, 58.e1.

20. Wyatt JS, Gluckman PD, Liu PY, et al.; CoolCap Study Group. Determinants of outcomes after head cooling for neonatal encephalopathy. Pediatrics 2007;119:912-21.

21. Gluckman PD, Wyatt JS, Azzopardi D, et al. Selective head cooling with mild systemic hypothermia after neonatal encephalopathy: multicentre randomised trial. Lancet 2005;365:663-70.

22. Eicher DJ, Wagner CL, Katikaneni LP, et al. Moderate hypothermia in neonatal encephalopathy: efficacy outcomes. Pediatr Neurol 2005;32:11-7.

23. Edwards RM, Naik-Mathuria BJ, Gay AN, Olutoye OO, Teruya J. Parameters of thromboelastography in healthy newborns. Am J Clin Pathol 2008; 130:99-102. 
24. Luddington RJ. Thrombelastography/thromboelastometry. Clin Lab Haematol 2005;27:81-90.

25. Scarpelini S, Rhind SG, Nascimento B, et al. Normal range values for thromboelastography in healthy adult volunteers. Braz J Med Biol Res 2009;42:1210-7.

26. Nair SC, Dargaud Y, Chitlur M, Srivastava A. Tests of global haemostasis and their applications in bleeding disorders. Haemophilia 2010;16:Suppl 5:85-92.

27. Haizinger B, Gombotz H, Rehak P, Geiselseder G, Mair R. Activated thrombelastogram in neonates and infants with complex congenital heart disease in comparison with healthy children. Br J Anaesth 2006;97:545-52.

28. Moganasundram S, Hunt BJ, Sykes K, et al. The relationship among thromboelastography, hemostatic variables, and bleeding after cardiopulmonary bypass surgery in children. Anesth Analg 2010;110:995-1002.

29. Alexander DC, Butt WW, Best JD, Donath SM, Monagle PT, Shekerdemian LS. Correlation of thromboelastography with standard tests of anticoagulation in paediatric patients receiving extracorporeal life support. Thromb Res 2010;125:387-92.

30. Kang Y, Borland LM, Picone J, Martin LK. Intraoperative coagulation changes in children undergoing liver transplantation. Anesthesiology 1989;71:44-7.
31. Goobie SM, Soriano SG, Zurakowski D, McGowan FX, Rockoff MA. Hemostatic changes in pediatric neurosurgical patients as evaluated by thrombelastograph. Anesth Analg 2001;93:887-92.

32. Chan KL, Summerhayes RG, Ignjatovic V, Horton SB, Monagle PT. Reference values for kaolin-activated thromboelastography in healthy children. Anesth Analg 2007;105:1610-3, table of contents.

33. Christensen RD, Sheffield MJ, Lambert DK, Baer VL. Effect of therapeutic hypothermia in neonates with hypoxic-ischemic encephalopathy on platelet function. Neonatology 2012;101:91-4.

34. Fraser CD Jr, Jaquiss RD, Rosenthal DN, et al.; Berlin Heart Study Investigators. Prospective trial of a pediatric ventricular assist device. $\mathrm{N}$ Engl J Med 2012;367:532-41.

35. Mitchell LG, Goldenberg NA, Male C, Kenet G, Monagle P, NowakGöttl U; Perinatal and Paediatric Haemostasis Subcommittee of the SSC of the ISTH. Definition of clinical efficacy and safety outcomes for clinical trials in deep venous thrombosis and pulmonary embolism in children. J Thromb Haemost 2011;9:1856-8.

36. Shinya H, Matsuo N, Takeyama N, Tanaka T. Hyperammonemia inhibits platelet aggregation in rats. Thromb Res 1996;81:195-201.

37. Polin R. Pathophysiology of metabolic disease of the liver. In: Gibson J, Berry G, eds. Fetal and Neonatal Physiology. Philadelphia, PA: W.B. Saunders Co, 2004:1211-8. 